Methods Anaesthetised C57BL6 mice were given an intratracheal $20 \mu \mathrm{g}$ dose of lipopolysaccharide (LPS). Mice were sacrificed at various time points, their lungs were removed, immersed in a buffer to fix and permeabilise cells, and a single cell suspension was produced by mechanical disruption. After incubation at $37^{\circ} \mathrm{C}$ the cell suspension was washed and centrifuged. Samples were stained at room temperature in the dark with antibodies to identify alveolar macrophages (AM) and epithelial cells (AEC), and to measure the intracellular levels of active phosphorylated forms of ERK, p38 and MK2. Finally the cells were washed and resuspended for flow cytometric analysis.

Results AM showed a rapid increase in levels of phosphorylated MK2 (see Abstract P251 Figure 1) as well as p38 and ERK on stimulation by intraalveolar LPS. As MK2 showed the most robust response, we determined its phosphorylation in AEC. Those AEC expressing low levels of surface ICAM-1 (likely type II pneumocytes) showed a pattern of increased MK-2 phosphorylation, while ICAM-1 high expressing AEC (type I pneumocytes) did not show a clear response to LPS stimulation.

Conclusion We have developed a method of investigating activation of individual cells within the lung using flow cytometry, in terms of intracellular MAPkinase phosphorylation. Our data show that AM are rapidly activated upon stimulation by intraalveolar LPS, whereas AEC show signs of delayed activation with type II pneumocytes more responsive than type I cells. Applying this technique to other models, in which alternative cell types and/or pathways may be involved, will enhance our understanding of cellular activation and interactions during acute lung injury.

\section{P252 A PHYSIOLOGICAL CHARACTERISATION OF INJURY, INFLAMMATION AND RESOLUTION IN MURINE ASPIRATION PNEUMONITIS}

doi:10.1136/thx.2010.151076.3

B V Patel, M R Wilson, M Takata. Imperial College London, London, UK

Introduction and Objectives Aspiration pneumonitis is an important risk factor for acute lung injury/acute respiratory distress syndrome (ALI/ARDS). ${ }^{1}$ Animals models of ALI should ideally mimic the human situation, although to date none fully reflect the complex pathophysiology involved. Our objective was to develop, optimise and characterise a model of acid aspiration that would closely resemble clinical ALI/ARDS including both inflammatory and recovery phases. While longer-term animal models of acid aspiration have been utilised, their clinical applicability has been limited by high mortality or use of unilateral pulmonary instillation ${ }^{2}$.

Methods C57Bl6 mice were anaesthetized, orotracheally catheterised and instilled with $75 \mu \mathrm{l}$ of $0.1 \mathrm{M}$ hydrochloric acid. They were recovered with $\mathrm{FiO}_{2}$ of 0.4 for $6 \mathrm{~h}$ and then placed in room air. At pre-determined time points they underwent tracheostomy, low tidal volume ventilation $(8 \mathrm{ml} / \mathrm{kg})$, and arterial catheter placement. Respiratory system mechanics and arterial oxygenation were determined, and following termination, bronchoalveolar lavage (BAL) was sampled for neutrophil infiltration and total protein concentration.

Results Following acid aspiration mice showed significant weight loss, decreased $\mathrm{PaO}_{2}: \mathrm{FiO}_{2}$ ratios and increased respiratory system elastance compared to untreated controls. There was significant pulmonary endothelial/epithelial leak as measured by BAL protein concentration, as well as substantial neutrophil influx. Changes in all of these parameters were transient, peaking on days 1-3 and then resolving to near baseline by day 10 (Abstract P252 Table 1).
Abstract P252 Table 1

\begin{tabular}{lllllll}
\hline & Control & Day 1 & Day 2 & Day 3 & Day 5 & Day 10 \\
\hline Weight Loss & - & $-7.0 \pm$ & $-13.3 \pm$ & $-13.3 \pm$ & $-7.3 \pm$ & $-2.9 \pm$ \\
$(\%)(\mathrm{N}=5-11)$ & & 2.3 & 4.9 & 4.0 & 2.5 & 1.9 \\
$\mathrm{PaO}_{2}: \mathrm{FiO}_{2}$ & $507 \pm$ & $162 \pm$ & $310 \pm 74^{*}$ & $158 \pm$ & $359 \pm$ & $538 \pm$ \\
$(\mathrm{N}=3-5)$ & 37 & $97^{* * *}$ & & $69^{* *}$ & 97 & 11 \\
Respiratory & $0.028 \pm$ & $0.061 \pm$ & $0.065 \pm$ & $0.035 \pm$ & $0.032 \pm$ & $0.027 \pm$ \\
Elastance & 0.002 & $0.018^{* * *}$ & $0.008^{* * *}$ & 0.003 & 0.002 & 0.001 \\
$\left(\mathrm{cmH}_{2} \mathrm{O} / \mu \mathrm{l}\right)$ & & & & & & \\
$(\mathrm{N}=3-4)$ & & & & & & \\
$\mathrm{BAL}$ Protein & $0.15 \pm$ & $4.86 \pm$ & $3.36 \pm$ & $1.72 \pm$ & $0.73 \pm$ & $0.31 \pm$ \\
Conc. $(\mathrm{mg} / \mathrm{ml})$ & 0.02 & $0.47^{* * *}$ & $0.56^{* * *}$ & $0.89^{* *}$ & 0.20 & 0.09 \\
$(\mathrm{~N}=3-6)$ & & & & & & \\
$\mathrm{BAL} \mathrm{Neutrophil}$ & $0.002 \pm$ & $5.7 \pm 3.8$ & $28.4 \pm$ & $6.60 \pm$ & $0.47 \pm$ & $0.003 \pm$ \\
Count $\left(\times 10^{4} / \mathrm{ml}\right)$ & 0.004 & & $5.1^{* * *}$ & 0.63 & 0.19 & 0.001 \\
$(\mathrm{~N}=3-4)$ & & & & & & \\
\hline
\end{tabular}

All timepoints compared with One-Way Analysis of Variance with Bonferroni test.

${ }^{* *} p<0.001,{ }^{* *} p<0.01,{ }^{*} p<0.05$ vs control.

Conclusion In this study, we describe a translational model of acid-induced lung injury that shows significant injury and inflammation resolving over a 10-day period. It shows many characteristics of clinical ALI/ARDS including severe hypoxaemia, worsening respiratory mechanics, alveolar-capillary barrier permeability, and neutrophilic infiltration within the alveolar space. This resolving model of aspiration pneumonitis may allow further elucidation of the pathophysiological mechanisms of ALI/ARDS and its resolution, in particular oedema formation and inflammatory cell recruitment.

Funding This work was supported by the Wellcome Trust and National Institute of Academic Anaesthesia.

\section{REFERENCES}

1. Rubenfeld, et al. N Engl J Med, 2005;353:1685-93

2. Amigoni, et al. Anesthesiology, 2008;108:1037-46.

\section{P253 PULMONARY INFILTRATES IN HIV NEGATIVE IMMUNOCOMPROMISED PATIENTS: OUTCOME AND PREDICTIVE FACTORS}

doi:10.1136/thx.2010.151076.4

S Chandramouli, M Devine, H Shoo, N Duffy. University Hospital Aintree, Liverpool, UK

Introduction The incidence of pulmonary complications in immunocompromised patients is on the rise mainly driven by increased organ and bone marrow transplants, more aggressive chemotherapy and novel immunosuppressants. ${ }^{1}$ Mortality rates in HIV negative immunosuppressed patients vary between $40-85 \% .{ }^{1-3}$ Prognostic factors have been previously described in these patients and these include need for mechanical ventilation and delay in diagnosis. ${ }^{2}$

Aims To describe the characteristics of immunocompromised HIV negative patients presenting with pulmonary infiltrates and to examine the factors that lead to increased mortality.

Methods All HIV negative immunocompromised patients who had bronchoalveolar (BAL) samples taken from January 2007 to January 2010 were identified from laboratory data. Their baseline demographics, immune status, clinical presentation, radiological picture and blood results at the time of BAL sampling were obtained from hospital records. The influence of various categorical variables on mortality was evaluated using Chi-square test. Logistic regression was then used to quantify the impact of each of these variables and obtain OR

Results 87 patients ( 33 women) with a median (range) age of 62 (21-87) years were studied. Overall mortality was 49\% (42 
patients). The Abstract P253 Table 1 shows the effect of different variables on mortality. This was not affected by sex, transplant status or laboratory parameters. With logistic regression analysis, we found that haematological malignancies (OR 7.44, 1.96-28.20), long term steroids (OR 7.87, 2.36-26.20) and age >65years (OR 3.94, 1.36-11.40) were significantly associated with mortality. The relationship with immunosuppressive drugs was less significant.

\section{Abstract P253 Table 1}

\begin{tabular}{llll}
\hline Variable & Died & Survived & Chi square \\
\hline Haematological malignancy & $21(70 \%)$ & $9(30 \%)$ & $7.37(\mathrm{p}=0.0006)$ \\
Long term steroids & $21(72 \%)$ & $8(28 \%)$ & $8.7(\mathrm{p}=0.0003)$ \\
Interstitial lung disease & $9(69 \%)$ & $4(31 \%)$ & $1.79(\mathrm{p}=0.18)$ \\
Connective tissue disease & $7(64 \%)$ & $4(36 \%)$ & $0.39(\mathrm{p}=0.44)$ \\
Recent immunosuppressants or chemo- & $30(59 \%)$ & $21(41 \%)$ & $3.7(\mathrm{p}=0.05)$ \\
therapy & $11(58 \%)$ & $8(42 \%)$ & $0.47(\mathrm{p}=0.49)$ \\
PCP PCR positive & $26(67 \%)$ & $12(33 \%)$ & $9.58(\mathrm{p}=0.002)$ \\
Age $>65$ years & & &
\end{tabular}

Conclusions HIV negative immunocompromised patients with pulmonary infiltrates are more likely to die if they have a haematological malignancy, are on long term steroid therapy or aged $>65$ years. The effect of immunosuppressive drugs needs more elucidation. Careful attention needs to be paid to these groups of patients to pick up early signs of deterioration.

\section{REFERENCES}

1. Shorr AF, et al. Chest 2004;125;260-71.

Ana Rañó, et al. Chest 2002;122;253-61

3. Robert H Poe, et al. Arch Intern Med 1986;146:1304-8.

4. Rubenfeld GD, et al. Ann Intern Med 1996;125:625-633.

\section{P254 INVESTIGATING HMGB1 AS A POTENTIAL INFLAMMATORY MEDIATOR IN BRAIN DEATH INDUCED LUNG DAMAGE}

doi:10.1136/thx.2010.151076.5

A E Vallance, H R Walden, D M Karamanou, C R Fox, A J Rostron, J A Kirby, A J Simpson, J H Dark, A J Fisher. Institute of Cellular Medicine, Newcastle University, Newcastle upon Tyne, UK

Background Donor lungs are particularly susceptible to the haemodynamic instability and systemic inflammation which occurs following induction of brain death (BD). Increased donor lung inflammation with IL-6 or IL-8 is associated with poor post-transplant outcomes. Donor LPS pre-conditioning significantly ameliorates the lung inflammation after $\mathrm{BD}$ in a rat model suggesting that TLR-4 ligation following BD contributes to donor lung inflammation, even in the absence of LPS. We hypothesised that HMGB1, an alarmin released from damaged tissue and immune cells and known TLR-4 ligand, may act as an inflammatory mediator in BD induced lung injury.

Methods BD was induced in a rat model using rapid intra-cranial balloon inflation and bronchoalveolar lavage (BAL), serum and lung tissue were collected and compared with sham operated controls rats. The HMGB1 concentration in the rat serum and BAL was measured by ELISA. Real-time PCR was used to assess HMGB1 mRNA expression in lung tissue following BD. HMGB1 immunolocalisation studies were performed on $\mathrm{BD}$ and sham rat lung tissue. Finally HMGB1 staining was assessed in lung tissue from human $\mathrm{BD}$ donors and normal controls.

Results The BAL HMGB1 concentration was significantly higher in the $\mathrm{BD}$ group $(965 \pm 302 \mathrm{ng} / \mathrm{ml})$ than in the sham group
$(655 \pm 274 \mathrm{ng} / \mathrm{ml})(\mathrm{p}=0.0172)$. There was however no difference in HMGB1 gene expression between the two groups. HMGB1 positive staining was visualised in a nuclear and extra-nuclear location and was dispersed throughout the rat and human lung with a greater density around the bronchioles. There was a significantly higher area of positive staining in $\mathrm{BD}$ rat lung tissue than sham tissue $(p=0.0345)$. No difference was seen in the human $\mathrm{BD}$ lungs compared to controls (Abstract P254 Figure 1).

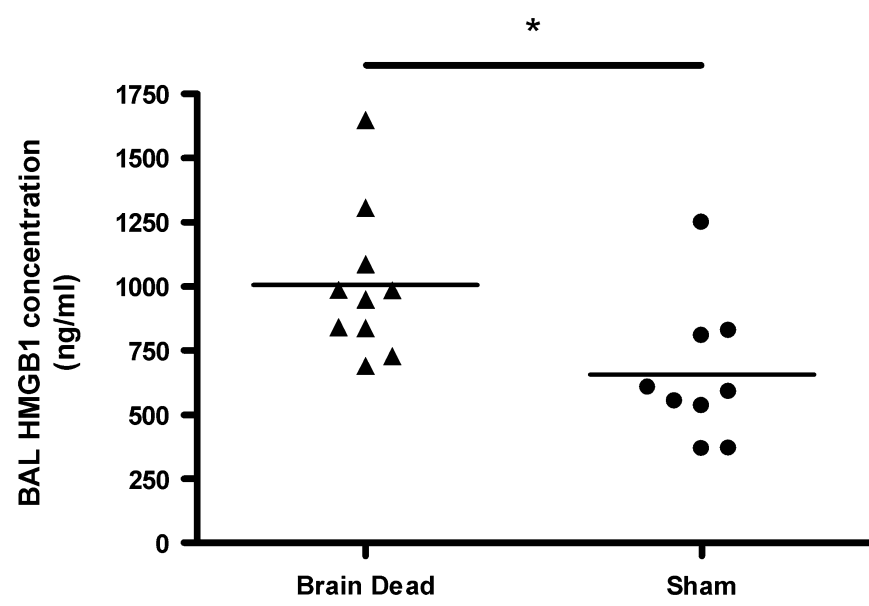

Abstract P254 Figure 1

Conclusions HMGB1 is likely to be released passively from lung cells suffering sustained damage during $\mathrm{BD}$. As HMGB1 gene transcription is unchanged up to $5 \mathrm{~h}$ following $\mathrm{BD}$, the lung tissue is most likely releasing presynthesised cellular HMGB1. This study provides evidence of the presence of an alarmin which is likely to potentiate inflammation in the donor lung via the TLR-4 pathway. If release occurs early following BD, HMGB1 could be an important initiating mediator in donor lung inflammation.

\section{Clinical studies in pulmonary embolism P255 DEFINING A CIRCADIAN PATTERN OF PRESENTATION OF PULMONARY EMBOLISM ON CTINO IMAGING}

doi:10.1136/thx.2010.151076.6

N Shah, A S Jubber, I Syed. Barking Havering and Redbridge NHS Trust, Romford, UK

Purpose To evaluate whether there is an association between the chronological occurrence of pulmonary emboli, anatomical site, radiological extent and associated clinical risk factors.

Materials and Methods 1410 consecutive CTPA and VQ scans performed between December 2005 and October 2008 were retrospectively reviewed independently by 2 thoracic radiologists $(\mathrm{kc}=0.78)$. A third observer was used in equivocal cases. 270 cases of pulmonary emboli were identified. Records were available for 180 patients. 40 were excluded on the basis of incomplete presenting details. All diagnosed cases of pulmonary emboli were risk stratified according to the Wells score, underlying co-morbidities including diabetes, DVT, malignancy and cardiomyopathy. Morbidity, mortality and survival data were recorded. For 140 (Age 67y SD $\pm 17,61$ male), the occurrence of the pulmonary emboli based on onset of symptoms was defined by four time intervals. Each group was subdivided according to extent of clot. The average Wells score was calculated for each $6 \mathrm{~h}$ period. 